relationships by Spearman'. Response to smoking cessation was determined by paired Wilcoxon.

Results Vastus lateralis protein carbonyls were related to klotho levels $(\mathrm{r}=0.34, \mathrm{p}=0.02)$. Patients with a low FFMI $(\mathrm{n}=12)$ had increased klotho levels compared to those who did not, $14.0(8.5,30.0)$ v $9.2(6.5,13.2) \mathrm{pg} / \mathrm{mg} ; \mathrm{p}=0.04$, however, protein carbonyls were not different $(\mathrm{p}=0.08)$. Conversely, patients with muscle weakness defined as a QMVC/BMI below $1.2(\mathrm{n}=24)$ had higher protein carbonylation, $0.76(0.52,0.99)$ v $0.51(0.40,0.71) \mathrm{nmol} / \mathrm{mg} ; \mathrm{p}=0.04$, but no difference in klotho (see figure 1). Patients with both a low FFMI and QMVC/ BMI $(n=6)$ had elevated levels of klotho and protein carbonyls ( $p=0.02$ and $p=0.03$ respectively). In those completing smoking cessation, protein carbonyls, tended to fall, $0.75(0.64,1.24)$ to $0.61(0.50,0.71) \mathrm{nmol} / \mathrm{mg} ; \mathrm{p}=0.08$, however, klotho levels did not change $(\mathrm{p}=0.38)$.

Conclusions Klotho levels are related to oxidative stress within the quadriceps. Klotho levels are unexpectedly higher in those with reduced FFMI without increased oxidative stress. However, when there is loss of muscle mass and strength, increased klotho levels in muscle are accompanied by increased oxidative stress. Klotho may have a protective role against chronic oxidative stress in the skeletal muscle of patients with a reduced FFMI.

\section{S52 MIR-181 INCREASES IN THE QUADRICEPS MUSCLE OF COPD PATIENTS AFTER AN ACUTE BOUT OF EXERCISE}

${ }^{1}$ AVJ Donaldson, ${ }^{1} \mathrm{RJ}$ Tanner, ${ }^{1} \mathrm{CA}$ Davey, ${ }^{1} \mathrm{~N}$ Hopkinson, 'W D-C Man, ${ }^{2} \mathrm{PR}$ Kemp, ${ }^{1}$ MI Polkey; ${ }^{1}$ NIHR Respiratory Biomedical Research Unit of Royal Brompton and Harefield NHS Foundation Trust and Imperial College, London, UK; ${ }^{2}$ Imperial College, London, UK

\subsection{6/thoraxjnl-2013-204457.59}

Introduction MicroRNAs (miRs) are small non-coding RNAs that regulate gene expression and have been implicated in the control of skeletal muscle phenotype. Our group has previously shown that muscle-specific miRs are altered, and correlate with physiological parameters in the quadriceps ${ }^{1}$ and plasma $^{2}$ of COPD patients. MiRs may therefore have a mechanistic role in the development of skeletal muscle dysfunction in COPD.

We hypothesised that muscle-specific miRs are involved in skeletal muscle adaptation to exercise and that changes in quadriceps miR expression after acute exercise would differ between patients and controls.

Methods 20 COPD patients and 11 controls were studied. Fasted quadriceps biopsies were taken before and one hour after an incremental, symptom limited, cycle exercise test. Muscle-specific miR-1,-499,-133, and -206 as well as miR-181, a more widely expressed miR associated with inflammatory responses, were quantified using qPCR. Paired analyses were performed using paired T-test or paired Wilcoxon.

Results See table of characteristics (Table 1)

Muscle miR results:

MiR-1, -499, -133 did not change with exercise. MiR-181 increased $\mathrm{x} 1.5$ in the COPD patients only one hour after exercise $(\mathrm{p}=0.017$, controls $\mathrm{p}=0.83)$. There was also a trend for miR-206 to increase in the COPD group only $(\mathrm{p}=0.07)$.

Conclusions Muscle-specific miRs do not change one hour after an acute bout of exercise in COPD patients or controls. However, miR-181 increased in the quadriceps of COPD patients. MiR-181 is not restricted to, but functions in muscle, and in addition has been shown to be linked to inflammatory signalling. The difference in the response of miR-181 expression to exercise is consistent with COPD patients having a greater inflammatory response to exercise stimulus than controls.

\section{REFERENCES}

1. Lewis A, et al Thorax 2012;67:26-34

2. Donaldson AVJ et al Thorax 2013: EPUB 28/06/2013

\begin{tabular}{|c|c|c|c|}
\hline \multicolumn{2}{|c|}{ Mean (SD) or Median (IQR) } & \multirow[b]{2}{*}{ Control } & \multirow{2}{*}{$\begin{array}{l}\begin{array}{l}\text { significance of } \\
\text { difference }\end{array} \\
\begin{array}{l}{ }^{*}(p<0.05) \\
{ }^{* *}(p=<0.01) \\
* * *(p=<0.001)\end{array}\end{array}$} \\
\hline & COPD & & \\
\hline Number & 20 & 11 & \\
\hline Male (female) & $13(7)$ & $6(5)$ & \\
\hline Age (years) & $65.9(9.1)$ & $62.8(7.2)$ & ns \\
\hline FFFMI $\left(\mathrm{kg} / \mathrm{m}^{2}\right)$ & $18.2(2.7)$ & $17.9(3.1)$ & ns \\
\hline FEV1 (\% pred) & $56.5(22.4)$ & $103.6(13.7)$ & \\
\hline Best MVC (kg) & $31.38(9.5)$ & $31.0(6.5)$ & ns \\
\hline average daily step count & $4721(6421,2453)$ & $9140(12635,5682)$ & ** \\
\hline Workload, (watt, \% pred) & $69.9(28.8)$ & $146(34.1)$ & $* * *$ \\
\hline $\begin{array}{l}\text { V'02 L/min max } \\
\text { exercise (\% pred) }\end{array}$ & $76.0(21.6)$ & $110.5(16.9)$ & $* * *$ \\
\hline
\end{tabular}

\section{S53 STUDYING FIBRE SPECIFIC GENE EXPRESSION IN COPD USING LASER CAPTURE MICRO-DISSECTION IN HUMAN SKELETAL MUSCLE}

${ }^{1} \mathrm{D}$ Mohan, ${ }^{2} \mathrm{~A}$ Lewis, ${ }^{1} \mathrm{MS}$ Patel, ${ }^{1} \mathrm{~K}$ Curtis, ${ }^{1} \mathrm{R}$ Tanner, ${ }^{2} \mathrm{P}$ Kemp, ${ }^{1} \mathrm{Ml}$ Polkey; ${ }^{1}$ NIHR Respiratory Biomedical Research Unit, Royal Brompton \& Harefield NHS Foundation Trust and Imperial College, London, UK; ${ }^{2}$ Section of Molecular Medicine, National Heart and Lung Institute, Imperial College London, London, UK

\subsection{6/thoraxjnl-2013-204457.60}

Background Approximately 30\% of patients with Chronic Obstructive Pulmonary Disease (COPD) exhibit peripheral skeletal muscle dysfunction and a shift towards type II glycolytic fibres in the quadriceps compared to healthy controls (Nanatek et al, 2013). Previous work to elucidate the molecular mechanisms underlying these changes has relied on whole biopsy samples and may have missed fibre-specific pathways; thus a method to evaluate fibre specific signalling pathways would be useful.

Objective To describe a novel laser capture micro-dissection (LCM) method to examine fibre-specific signalling in quadriceps biopsies.

Methods First larger Intercostal muscle biopsies were used to validate the methodology since they yielded more RNA. Fibres were classified as type- 2 positive or type- 2 negative based on immunoreactivity with a type- 2 fibre specific anti-myosin Heavy Chain Alexa FLUOR 488 antibody. The type- 2 negative fibre population was hence assumed to contain type- 1 fibres, which was confirmed by the type- 2 negative fibre population exhibiting a higher myhc7/2 mRNA ratio and expressing higher levels of genes associated with type- 1 fibres, e.g. TNNT-1 and STARS, and lower levels of genes associated with type- 2 fibres, e.g. TGF-B, myostatin, GAPDH and HDAC-4 $(\mathrm{n}=2)$.

We then examined OCT-embedded vastus lateralis muscle biopsy specimens. 10micron cryosections underwent fixation with 4\% paraformaldehyde before immunostaining. LCM (PALM Microbeam, Zeiss, UK) was used to capture type I and type II fibre populations, before RNA extraction with RNAeasy FFPE kit (Qiagen, USA) and rtPCR to obtain cDNA. Sybr-II 